\title{
The Fellows' Pelvic Research Network (FPRN) 5 years later: an update
}

\author{
Lior Lowenstein • Vivian Sung • Stephen Young • \\ Joseph Schaffer
}

Published online: 24 August 2012

(C) The International Urogynecological Association 2012

\section{Introduction}

Five years ago an initiative was launched during the meeting of the American Urogynecologic Society (AUGS) by the president of the Society of Gynecological Surgeons (SGS), the head of the research committee of the SGS, and a fellow of the society: to establish a research network for fellows in urogynecology and female urology. The agenda of the network was published in this journal [1] and included the following objectives: "Create an environment for fellows to participate in collaborative research and conduct multicenter studies as primary investigators". The main purpose of the network was to enhance fellows' knowledge and skills in study design, implementation of multicenter studies, data management, and statistical analysis. It was also hoped that the network would provide an environment for fellows to develop long-term professional relationships.

L. Lowenstein $(\bowtie)$

Department of Obstetrics and Gynecology, Rambam Health Care Campus, Ruth and Bruce Rappaport Faculty of Medicine

Technion-Israel Institute of Technology,

Haifa, Israel

e-mail: 1_lior@rambam.health.gov.il

\section{Sung}

The Division of Urogynecology and Reconstructive Pelvic Surgery, Department of Obstetrics and Gynecology,

Alpert Medical School of Brown University,

Providence, RI, USA

\section{S. Young}

Dept Ob/Gyn,

University of Massachusetts Memorial Medical Center,

Worcester, MA, USA

\section{J. Schaffer}

Division of Female Pelvic Medicine and Reconstructive Surgery, University of Texas Southwestern Medical Center,

Dallas, TX, USA
This initiative was inspired by acknowledgement of major differences in clinical research and mentoring opportunities for fellows from different programs throughout the USA. In addition to didactic research training for fellows, the network was envisioned to enable the conduct of large scale studies with participants from across the USA. This was believed to be particularly important for the investigation of rare conditions and regional differences in clinical practice and outcomes.

We modeled this new network on the two National Institutes of Health (NIH)-sponsored multicenter clinical trial networks in urogynecology that were being conducted in the USA at that time: (1) the Urinary Incontinence Treatment Network, which included urologists and urogynecologists from nine centers across the country; and (2) the Pelvic Floor Disorders Network, which included seven centers. Our network differed from these NIH networks in two main ways: (a) we operate on a very low budget, which is allocated by the SGS, (b) we have no restrictions or limitations on initial membership. Participation in the network was extended to all fellows in female urology and urogynecology programs in the USA.

\section{First scientific meeting}

On April 2007, 18 fellows from 18 different urogynecology and female urology programs across the USA attended the first meeting of the Fellows' Pelvic Research Network (FPRN) at the annual SGS scientific conference. An advisory board consisting of six senior urogynecologists - a subgroup of the SGS Research Committee - volunteered to mentor the fellows and assist in establishment of the network. Three fellows were elected to the FPRN Steering Committee, in the roles of chair, secretary, and treasurer. At the first meeting two multicenter clinical research trials were chosen as the first projects. These were selected from nine projects that had been submitted by fellows. One of the advisory board members was selected to oversee the 
investigations. Changes in protocols and preparation of the manual of operation were among the first tasks. Once the manual of operation was approved by the group, a protocol was submitted to the institutional review board (IRB) by all members to the local committee of each fellow's institution. Conference calls were conducted on a regular basis. Accumulated data were submitted to the principal investigator and analyzed with the help of a statistician.

To increase fellows' awareness of the network, and to recruit more members, the FPRN presented a link on the SGS website (http://www.sgsonline.org/fprn.php), detailing the activities of the FPRN, policies and procedures, lists of currently active members and advisory board members, and an electronic membership application form. The decision was made not to limit the number of fellows participating in the network from any given site. However, all members at a site are expected to actively participate in meeting the recruitment goals for their site.

The FPRN holds bi-annual meetings. Each time, the agenda includes: an update about ongoing research projects, problem solving related to current studies, and presentation of new research ideas. The didactic portion of the meeting includes a guest lecturer from the field of urogynecology who is invited to present a state-of-the-art lecture.

\section{Second scientific meeting}

The second meeting was an even bigger success than the first. The number of attendees more than doubled to 43 members from 26 different sites. An update of the research projects was presented, and a third multicenter study was selected by the fellows and the advisory board for implementation by the network.

\section{Grant application}

In 2010, support from the NIH, National Institute of Diabetes and Digestive and Kidney Diseases was received in the form of an R13 award to help fund the September 2010 FPRN meeting (R13DK084687). These funds were used to offset travel expenses for fellows in Female Urology and in Female Pelvic Medicine and Reconstructive Pelvic Surgery, as well as Junior Advisory Board members and one invited speaker. As a direct result of this funding support, the number of Female Urology fellow members of the FPRN increased.

\section{Recent update on the steady growth of the network}

As of August 2011 the FPRN membership comprised 80 urogynecology and female urology fellows representing 45 programs across the USA. There are 8 Junior Advisory
Board members, 12 Senior Advisory Board members, and 2 Ad Hoc Advisory Board members. The possibility of expanding the network and opening membership to fellows from outside the USA and to those in minimally invasive fellowship programs was recently discussed. Both FPRN members and the advisory board agreed that such expansion would serve the interests of the network, provided that the new members take an active part in its activities.

\section{Research achievements}

From the perspective of research, the network has proved very productive. Over the past 3 years the network has published eight papers in peer-reviewed journals [1-8], and a further three manuscripts have been submitted for consideration for publication. In addition, the members of the network have actively participated in scientific conferences and presented nine of their studies as podium and poster presentations. In the year 2010 a retrospective multicenter study on outcomes after midurethral polypropylene sling (MUS) revision for voiding dysfunction was awarded the SGS presidential prize for outstanding research in gynecologic surgery. Currently, there are ten active ongoing research projects.

\section{Conclusion}

The FPRN has proved to be a successful, vibrant network for fellows in urogynecology and female urology from all over the USA. The research and didactic missions have been accomplished. The FPRN enables fellows from different parts of the country to work together on common projects and to develop personal as well as professional relationships. This has been extremely important for future collaborations among colleagues from the same field.

What about the future? Even the authors who were involved in establishing this network did not believe that it would achieve so much in 5 years. Our conservative prediction for the future is that the network will continue to grow and include new members from Canada as well as fellows in minimally invasive surgery.

We hope to increase funding for the network through submission of competitive grants. This would allow the network to undertake a variety of studies including prospective randomized control trials. To achieve this goal the collaboration of attending physicians from the participating sites will be necessary.

As multicenter studies have become increasingly common we hope that the FPRN will seed the opportunities for more research networks of attending physicians who have graduated from the FPRN. 
Conflicts of interest None.

\section{References}

1. Lowenstein L, Olivera C, Harvie H, Schaffer J, Young S (2008) The Fellows' Pelvic Research Network (FPRN). Int Urogynecol J Pelvic Floor Dysfunct 19(7):895-897

2. Sanses TV, Shahryarinejad A, Molden S, Hoskey KA, Abbasy S, Patterson D, Saks EK, Weber Lebrun EE, Gamble TL, King VG, Nguyen AL, Abed H, Young SB (2009) Anatomic outcomes of vaginal mesh procedure (Prolift, Gynecare) compared to uterosacral ligament suspension and abdominal sacrocolpopexy for pelvic organ prolapsed. Am J Obstet Gynecol 201 (5):519.e1-519.e8

3. Lowenstein L, Gamble T, Sanses TV, van Raalte H, Carberry C, Jakus S, Kambiss S, McAchran S, Pham T, Aschkenazi S, Hoskey K, Kenton K (2009) Sexual function is related to body image perception in women with pelvic organ prolapsed. J Sex Med 6(8):2286-2291
4. Schimpf MO, Harvie HS, Omotosho TB, Epstein LB, Jean-Michel M, Olivera CK, Rooney KE, Balgobin S, Ibeanu OA, Gala RB, Rogers RG (2010) Does vaginal size impact sexual activity and function? Int Urogynecol J Pelvic Floor Dysfunct 21(4):447-452

5. Lowenstein L, Gamble T, Sanses TV, van Raalte H, Carberry C, Jakus S, Kambiss S, McAchran S, Pham T, Aschkenazi S, Hoskey K, Kenton K (2009) Changes in sexual function after treatment for prolapse are related to the improvement in body image perception. J Sex Med 6(8):2286-2291

6. Molden S, Patterson D, Tarr M, Sanses T, Bracken J, Nguyen A, Harvie HS, White A, Hammil SA, Murphy M, Rogers RG (2010) Risk factors leading to midurethral sling revision: a multicenter case-control study. Int Urogynecol J Pelvic Floor Dysfunct 21(10):1253-1259

7. Molden S, Bracken J, Nguyen A, Harvie HS, Whit A, Hammil SL, Patterson D, Tarr M, Sanses T, Murphy M, Rogers RG (2010) A retrospective multicenter study on outcomes after midurethral polypropylene sling (MUS) revision for voiding dysfunction. J Female Pelvic Med Reconstr Surg 16(6):340-344

8. Harvie H, Lowenstein L, Omotosho T, Sanses T, Molden S, Hardy J, Brubaker L (2010) IRB variability in multicenter studies. J Female Pelvic Med Reconstr Surg 16(2):S12-S13 